This item was submitted to Loughborough's Research Repository by the author.

Items in Figshare are protected by copyright, with all rights reserved, unless otherwise indicated.

\title{
Information retrieval from civil engineering repositories: the importance of context and granularity
}

PLEASE CITE THE PUBLISHED VERSION

http://dx.doi.org/10.1061/(ASCE)CP.1943-5487.0000229

PUBLISHER

(C) American Society of Civil Engineers

VERSION

AM (Accepted Manuscript)

LICENCE

CC BY-NC-ND 4.0

\section{REPOSITORY RECORD}

Demian, Peter, and Panagiotis Balatsoukas. 2019. "Information Retrieval from Civil Engineering Repositories: The Importance of Context and Granularity”. figshare. https://hdl.handle.net/2134/11038. 
This item was submitted to Loughborough's Institutional Repository (https://dspace.lboro.ac.uk/) by the author and is made available under the following Creative Commons Licence conditions.

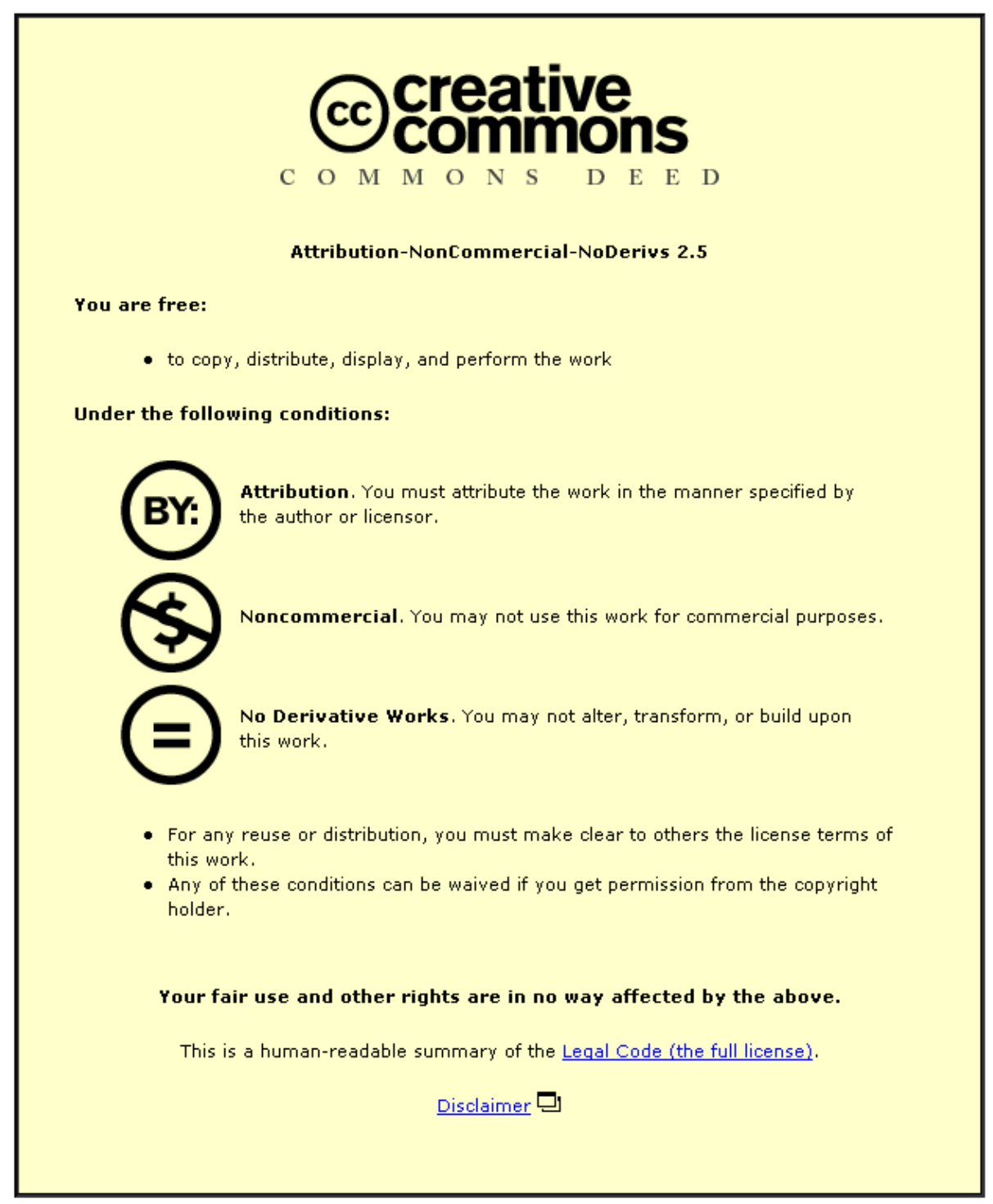

For the full text of this licence, please go to: http://creativecommons.org/licenses/by-nc-nd/2.5/ 


\title{
Information Retrieval from Civil Engineering \\ Repositories: the Importance of Context and \\ Granularity
}

\author{
Peter Demian ${ }^{1}$ and Panos Balatsoukas ${ }^{2}$ \\ 1 Lecturer in Construction Management, Loughborough University \\ 2 Research Fellow, University of Strathclyde
}

\begin{abstract}
Information about the design and construction of buildings can be structured in a particular way. This is especially correct given the increasing complexity of building product models and the emergence of Building Information Models with project documents linked to them. In addition, engineers usually have distinct information needs. Research shows that engineers working with Building Information Models place particular importance on the understanding of retrieved content before using it or applying it, and that exploration of context is essential for this understanding. Both these factors (the nature of engineering content and the information needs of engineers) make general information retrieval techniques for computing relevance and visualizing search results less applicable in civil engineering information retrieval systems. This paper argues that granularity is a fundamental concept that needs to be considered when measuring relevance and visualizing search results in information retrieval systems for repositories of building design and construction content. It is hypothesized that the design of systems with careful regard for granularity would improve engineers' relevance judgment behaviour. In order to test this hypothesis, a prototype system, called CoMem-XML, was developed and evaluated in terms of the time needed for users to find relevant information, the
\end{abstract}


Demian\&d Balatsoukas, Ms. No. CPENG-318 resubmitted to JCCE, September 2010

accuracy of their relevance judgment and their subjective satisfaction with the

prototype. A user study was conducted where test subjects were asked to complete tasks using various forms of the prototype, to complete a satisfaction questionnaire, and to be interviewed. The findings show that users perform better and are more satisfied when the search result interface of the CoMem-XML system presents only relevant information in context. On the other hand, interfaces that present the retrieved information out of context (i.e. without highlighting its position in the parts hierarchy) are less effective for participants to judge relevance.

\section{Keywords}

Information retrieval, information management, knowledge management, search results, Building Information Modelling

\section{Introduction}

Advances in information technology allow us to store and access more and more information. This information ranges from documents to structured data in relational databases through to semi-structured or unstructured data. With vast volumes of information becoming available, the problem of "information overload" is increasingly recognized and documented (Edmunds and Morris 2000, Lyman and Varian 2003). Repositories of civil engineering content have also grown in line with this general trend. In construction, the sheer quantity and heterogeneity of content in engineering applications has been recognized (KIM 2006, Rezgui 2001, Hicks et al. 2008). For example, in the UK construction industry on average one CAD document is produced for every $9 \mathrm{~m}^{2}$ of building floor space (Gray and Hughes 2001). The recent emergence of Building Information Modelling (BIM) gives additional motivation for this research. As the scope of building models continues to widen, it is becoming increasingly necessary to link external documents to models, a topical challenge 
Demian\&d Balatsoukas, Ms. No. CPENG-318 resubmitted to JCCE, September 2010

currently facing the industry. The professional press (Howell and Batcheler 2010) and online forums (Linkedln 2010) are ablaze with discussions of linking external data to CAD/BIM models.

The aim of this research is to investigate how knowledge management systems used by civil engineers and construction professionals can be tailored to facilitate the retrieval of relevant content and its effective use/reuse or application to the task at hand. This research focuses particularly on information and documents linked to buildings and building models, rather than the knowledge embedded in the design and construction processes and the professionals performing those processes (for example, Table 3,2 in Tan et al 2010 attempts to list the sources of construction project knowledge).

Engineering applications, particularly civil engineering and construction, possess two distinctive characteristics: firstly, construction content (such as Building Information Models) is more (or differently) structured than general repositories of documents; secondly, engineers and construction professionals have particular information needs and information-seeking habits, in contrast to general users of information systems. Both these factors make general information retrieval techniques for computing relevance and visualizing search results less applicable in civil engineering information retrieval systems and necessitate a bespoke solution.

This paper argues that granularity is a fundamental concept that needs to be considered when measuring relevance and visualizing search results in information retrieval systems for repositories of building design and construction content. Granularity is a novel concept for organizing and presenting information in search result interfaces of query-driven information retrieval systems in a manner that can 
Demian\&d Balatsoukas, Ms. No. CPENG-318 resubmitted to JCCE, September 2010

support understanding and exploration of the context of the retrieved information (for example, by highlighting its position in the parts hierarchy and exposing its relationship with relatives in the hierarchy). It is hypothesized that the design of systems with careful regard for granularity would improve engineers' relevance judgment performance. This research builds on past research by Demian and Fruchter (2006a, 2006b) on design reuse. Whereas the previous research applies information visualisation techniques to visualise an entire repository of design content and leaves the user to interact with this content, the present study focuses on querydriven systems. It investigates the display of search results from queries, examining how ranking and the inherent structure of the information can be exploited to optimise the user's relevance judgements and retrieval performance.

This paper is structured as follows. The following section reviews related research on the nature and structure of construction content, including Building Information Models and repositories of construction documents. The next section reviews related research on the information needs and information-seeking behaviour of engineers and construction practitioners. The next section reviews related research on the notion of granularity from information retrieval literature and discusses its application to construction search result interfaces. The next section describes the research method applied here: prototyping and user-testing of the prototype to test the underlying hypothesis: that granularity holds the key to improving the effectiveness of search result interfaces of construction knowledge management systems. The subsequent section describes the prototype search system whose search result interface exploits granularity. The Results section presents the results of the user tests. The final two sections discuss the results and draw final conclusions. 
Demian\&d Balatsoukas, Ms. No. CPENG-318 resubmitted to JCCE, September 2010

\section{Related Research: Construction Content}

The sheer quantity and heterogeneity of content in engineering applications (and particularly in construction) has already been noted in the previous section. The construction industry is following the universal trend of increasing volumes of information and digital content. In project-based contexts such as construction, information retrieval and knowledge management are particularly important to ensure that "wheels are not reinvented" and mistakes not repeated from project to project. Much of this "knowledge" is embedded in text and CAD documents generated during the design and construction phases (Anumba et al 2005, Shen et al 2009). The Extensible Markup Language (XML), used in this research, has also been considered by Agdas and Ellis (2010) for wide-ranging use in the construction industry.

Information retrieval techniques have been used in civil engineering to retrieve reusable designs (Demian and Fruchter 2005) and to retrieve contextual information from past projects to improve the accuracy of future cost estimates (Kiziltas and Akinci 2010). Granularity, central to this research, is tangentially addressed by Kovacevic et al,. (2008) whose system identifies specific paragraphs when retrieving web pages which answer particular construction-related questions. Beyond text, Brilakis and Soibelman (2008) automatically identify particular features in construction site photographs with a view subsequently to using information retrieval techniques to manage photograph collections. Bridging textual and geometric content, Caldas et al (2002) propose techniques for automatically classifying construction documents based on project CAD components. Lin and Soibelman (2009) augment standard information retrieval techniques with formal representations of domain knowledge to improve the performance of a search engine for online product information. Rezgui (2006) similarly uses domain knowledge, this time to formulate an ontology which informs the indexing and retrieval of construction 
Demian\&d Balatsoukas, Ms. No. CPENG-318 resubmitted to JCCE, September 2010

content.

The state of the art in digital content management in building design and construction projects is being transformed by the emergence of Building Information Modeling (Eastman et al. 2008). Whereas CAD models classically attempted to model the geometry of buildings or building components in two or three dimensions (Eastman 1999), Building Information Models attempt in addition to model non-geometric content. This content includes non-geometric attributes of physical building components (e.g. cost, Shen and Issa 2010) as well as non-geometric entities. For example, Building Information Models can include entities to model the processes of design (Austin et al 2000) and construction (Koo and Fischer 2000), as well as the organizations (teams and individuals) that execute activities in those processes (Kunz et al 1998). In addition, Building Information Modeling is not limited to the design and construction phases but is extending to cover the whole life cycle of constructed facilities, from briefing/programming and early design concepts, through to facilities management and even disposal of buildings.

\section{Related Research: Information-Seeking Behaviour of Engineers}

Early studies on the information-seeking behaviour of engineers by Allen (1977) and Gerstberger and Allen (1968) show that the information sources used by engineers differed across the various stages of the product development cycle (for example, from written to oral communication and from formal to informal resources). Similar were the findings of Chakrabarti et al. (1983), Pinelli et al (1993), Leckie et al (1996), Ellis and Haugan (1997), and Fidel and Green (2004) who also conclude that engineers use information resources that can be easily accessible. A detailed review of other criteria considered when selecting resources is given by Fidel and Green (2004). Even within engineers as information users, Kwasitsu (2003) found 
Demian\&d Balatsoukas, Ms. No. CPENG-318 resubmitted to JCCE, September 2010

that differences exist depending on an engineer's job role and level of education. In addition, these studies show that engineers prefer the use of informal information and communication channels such as company reports and colleagues, rather than academic resources, such as scholarly papers and textbooks.

Hertzum and Pejtersen (2000) use case studies to examine the way engineers utilize informal information sources, such as company reports and colleagues. They find that engineers rely on colleagues and oral communication to provide relevant information. This is because engineers feel that interpersonal contact provides information about the background and context related to the information needed. Such context is not always provided by repositories. Other barriers to using written information sources include cost, time, the availability of information, the usability of tools and the amount of intellectual effort needed in searching and navigating.

Demian and Fruchter (2006a) in an ethnographic study highlight the critical role of understanding in the information-seeking behaviour of civil engineers and construction managers. In this manner, the effective acquisition and reuse of information from both internal and external resources requires a significant amount of understanding of its context. Demian and Fruchter, and earlier Hertzum and Pejtersen (2000), argue that the process of understanding is more difficult to achieve in the case of external information sources such as knowledge management systems and corporate databases rather than internal resources (such as personal memory). Many of these external systems do not provide contextual information to aid the understanding of retrieved items. The research reported in this paper is a step towards this end. Other factors that can impede understanding of external information include engineers' unfamiliarity with the contents of a repository and lack of training (Ye and Fischer, 2002). 
Demian\&d Balatsoukas, Ms. No. CPENG-318 resubmitted to JCCE, September 2010

In addition to information-seeking processes, recent studies have also investigated the effects of interface components (such as web pages, web search engines and digital libraries of corporate information). Kraaijenbrick (2007) examines the problems encountered by engineers while searching for information on the web for new product development (NPD). The findings show that engineers expect to browse tree-based hierarchical information systems both downwards and upwards in order to understand and explore the context of a specific information item.

In another study, Demian and Fruchter (2006b) provide a more detailed account of the interaction of engineers with information systems. In particular, the purpose of that study was to examine the performance and satisfaction of civil engineers with three systems that provided access to information about completed construction projects. The three systems differed only in the way search results are presented to users; the underlying repository being searched was the same. The CoMem system applied information visualisation techniques in order to display relevant information. Users of this system were given a visual overview of the entire repository. When relevant information is identified by the user from this overview, the context of this item can be explored in two interfaces that (1) identify and display related information in the hierarchy and (2) visualise the evolution history of that item. CoMem was compared to two other interfaces designed to represent more traditional tools:

Outline Tree modelled after Windows Explorer, and the HitList modelled after a textbased web search engine. The findings of a usability study showed that the Outline Tree was more time efficient for the completion of fact finding tasks, where the user is looking for a specific item of information. The researchers argued that this was because information was displayed in a small space and the value of the information scent (Pirolli et al., 2003) communicated by the upper branches of the hierarchy to 
Demian\&d Balatsoukas, Ms. No. CPENG-318 resubmitted to JCCE, September 2010

users was stronger. However, participants were able to complete exploratory tasks in less time using the ComMem prototype, and generally reported feeling more satisfied with CoMem. CoMem enabled designers to explore an item's context, thus supporting a better understanding of the item's relevance to an information need. Finally, although the HitList interface provided instant access to information, similar to search engines such as Google, it did not provide enough information about the context of the retrieved information. The HitList was the least time efficient system and participants were significantly less satisfied with this interface than the Outline Tree and CoMem interfaces.

Outside the domain of engineering, Vegas et al (2007) uses a 2D DocBall visualisation to show where relevant information occurs within a single web-page or website. The DocBall metaphor was incorporated in the design of a prototype search engine, called Bubble. In a user evaluation, participants found it easier to locate relevant information using the Bubble system than the baseline system (which represents a standard text-based search engine). The participants explained that in Bubble, information was displayed in context.

To summarise, the literature on the information-seeking behaviour of engineers shows that the exploration of context is important in the relevance judgment performance of engineers. Therefore, systems that store and provide access to information about building construction projects should not only provide a ranked list of results, but also should enhance results with information about the context and granularity of the retrieved information, such as the position of a building component in the design development life-cycle or its position in the overall building parts hierarchy. 
Demian\&d Balatsoukas, Ms. No. CPENG-318 resubmitted to JCCE, September 2010

\section{Related Research: Granularity}

As noted above, engineers are especially interested in understanding the context of the retrieved information in order to gain orientation and inspiration (Kraaijenbrick, 2007). This preference can be attributed to the highly structured and hierarchical nature of the building models with which engineers interact in order to find and reuse information. In the case of querying repositories of building content, context can be defined in terms of the relationships between a piece of information and other related information components. These relationships often take the form of the "is a part of" hierarchical relationship in a parts hierarchy of buildings and building components. In other words, in our case context is closely linked to granularity.

Granularity is a novel concept for organising and presenting information in search result interfaces of query-driven information retrieval systems in a manner that supports understanding and exploration of context. Although granularity has its roots in relational database technology, the modern concept of granularity stems from object-oriented programming and can be defined as "the logical size, structure and the extent to which a resource is used as part of another resource or contains parts of other resources" (Wu, 2005). More granular digital resources are larger and are composed of smaller pieces. Early research in large scale knowledge bases has made use of granularity for the semantic representation and retrieval of domain specific knowledge. For example, Wachsmuth and Meyer-Fujara (1990) made use of granularity in a medical knowledge base as a means of retrieving relevant packages of knowledge elements. A knowledge package could be related to a specific medical condition (e.g. hypertension) and each package could contain knowledge elements more specific to that condition. The granular representation of knowledge permitted structured and semantic-dependent access to packages and other associative knowledge elements following dynamic and static conditions. In our case, a 
Demian\&d Balatsoukas, Ms. No. CPENG-318 resubmitted to JCCE, September 2010

repository of construction content is divided into different projects (different buildings) with each building subdivided into subsystems (e.g. structural frame, ductwork) and each subsystem subdivided into components (a particular beam or column). In information retrieval research, the Extensible Markup Language (XML) has become the premier language for expressing the context and structure of information at various levels of granularity. Although mainstream information retrieval research, such as INEX (INitiative for the Evaluation of XML), has examined the effectiveness of different algorithms for the retrieval of information from collections of $\mathrm{XML}$ documents (INEX, 2009), there are few studies examining the effects of granularity of search result interfaces on user relevance judgment behaviour (Larsen et al., 2006; Pharo, 2008). For example, Larsen et al. (2006) found that participants at INEX experiments preferred to view whole documents as opposed to individual parts of documents when evaluating retrieved lists of articles from the IEEE Computer Society. The researchers suggested that this happened either because the title and initial metadata of the articles were more informative than the individual subparts of the articles (e.g. sections, subsections, sub-subsections), or because participants preferred to access the full article as opposed a part of it. A more detailed description of participants' behaviour at INEX was reported by Pharo (2008) who analysed data from 218 search sessions performed by 73 participants using the XML based Daffodil system. Daffodil is a Java-enabled application that indexes and provides access to collections of articles from two main digital libraries, (1) Wikipedia and (2) the IEEE Computer Society's repository of journal papers in computer science. Daffodil presents a list of relevant document elements or subparts as a response to a user's query (e.g. top level article, metadata, section, subsection level 1, subsection level 2, back matters) presented within the documents they belong to (Figure 1) (Malik et al., 2006 gives a review). 
Demian\&d Balatsoukas, Ms. No. CPENG-318 resubmitted to JCCE, September 2010

After a query was executed, participants were asked to rate the relevance of any retrieved document component (e.g.sub-subsection, subsection, section or whole document) based on a 5-point scale developed by Pechevski (2006). The five points on the scale were:

- Relevant but too broad (the retrieved document component contains relevant information but also includes other irrelevant information).

- Exactly Relevant answer (highly relevant information at the exact level).

- Relevant but too narrow (the retrieved document component contains relevant information which is usually accompanied with little context).

- Partial answer (the document component contains partially relevant information).

- Not relevant (the document component includes no relevant information).

It is noteworthy that relevance assessments before the iTrack 2006 were based on a three-point scale originated at interactive Text Retrieval Conference (TREC) (Relevant, Partially relevant and Not relevant). However, as opposed to traditional experiments at the TREC conference, the retrieval of information from $\mathrm{XML}$ documents cannot be made solely in terms of topicality (for example, whether the contents of the documents are relevant or not relevant to a user's need). Another dimension that should be included in any relevance judgment is context, inferred from the parent-child relationship in the hierarchy.

Pharo (2008) found that participants preferred to assess the relevance of sections and subsections as opposed to full documents (i.e. as opposed to the article level). However, both studies by Larsen et al (2006) and Pharo (2008) concluded that a balance must be struck between too coarse and too fine granularity. Document 
Demian\&d Balatsoukas, Ms. No. CPENG-318 resubmitted to JCCE, September 2010

components of too fine granularity (especially sub-subsections) were used less frequently by participants to judge relevance because they do not provide enough context. In addition, it was found that participants preferred to view general details about the whole of the retrieved document (i.e. metadata elements and document title) in the search result interface but they found it useful to explore document elements (i.e. sections and subsections) when accessing the full text document.

Although INEX constitutes the main initiative for investigating user interaction with the retrieval of document components at different levels of granularity, Lehtonen (2006) criticised its focus on specific types of collections, the ongoing debate as to whether users prefer to interact with document components at specific levels of granularity and the lack of contextual hints as a means for users to formulate queries. Lehtonen argues that these issues can impede the generalisability of the findings of user studies in XML information retrieval. The present study addresses some of these limitations by focusing on a different situation (i.e. civil and building engineers searching for information in an XML repository of construction projects) and the use of both structural (elements that define the structure of the content, such as components and sub-components) and contextual (elements that define the meaning of content, such as legislation or maintenance cost) metadata in order to encode the content of the projects included in the collection. However, this study retains the interest in the investigation of the effects of granularity on user performance and satisfaction during the relevance judgment process. Furthermore our research updates the findings of earlier studies on information retrieval in the context of domain-specific knowledge bases (e.g. Watchmuth, 1990) by employing web-based semantic technologies (i.e. $\mathrm{XML}$ ) in the study of the effects of granularity on relevance judgment behavior and following standard INEX methodology.

Some studies outside of INEX have tried to investigate user interaction with different 
Demian\&d Balatsoukas, Ms. No. CPENG-318 resubmitted to JCCE, September 2010

types of interfaces (Hammer-Abebi et al., 2006) as well as the use of different data collection methods (such as interviews) (Betsi et al, 2006). Hammer-Aebi et al (2006) investigate the effects of two different interfaces for displaying document components on relevance judgment behaviour. The two interfaces were: (1) The Context interface and (2) The Isolated interface. The former displays relevant document components in their original context, while the latter presents each component isolated from its context. It was found that participants tended to use the individual document components as opposed to whole documents to judge relevance. However, no significant differences were observed between the two interfaces in terms of time needed to find relevant information or the preferred level of granularity for judging relevance.

Betsi et al (2006) performed semi-structured interviews with 10 software engineers in order to investigate users' perceptions about the advantages and disadvantages of $\mathrm{XML}$ information retrieval for information searching. They report that participants found the retrieval of document sections more useful than the full document. However, information about the whole document was important in order to provide context to the retrieved subparts (i.e. document components). Users also wanted to see and understand parent-child relationships in the hierarchy. They indicated that the search result interface should also display metadata to help users understand and evaluate the retrieved information.

\section{Method}

In order to address the aim of this study a user study was conducted to investigate participants' interaction with different displays of search results. Prototype interfaces were developed and test participants recruited and asked to complete tasks using the prototype tools. Interaction with the tools was observed. The order of use of the 
Demian\&d Balatsoukas, Ms. No. CPENG-318 resubmitted to JCCE, September 2010

three prototypes was randomized to minimize the effects of learning and familiarity with the tasks or data. After executing the tasks, each participant was asked to complete a post-test satisfaction questionnaire and participate in a semi-structured interview.

A total of 12 staff and students of the Department of Civil and Building Engineering at Loughborough University volunteered to participate in the study in response to a circular email invitation to the whole Department. All participants were frequent users of electronic information services and the World Wide Web. All candidate participants were contacted individually and asked to fill in a screening questionnaire in advance. The purpose of this questionnaire was to ensure that all participants were frequent users of electronic information services and to gather some demographic data. Selection of participants was limited to experienced web users only, this group being more motivated to perform the tasks than novice or inexperienced users of the web (Rubin and Chisnell, 2008). The number and composition of participants reflects the number of initial volunteers who passed the pre-screening. The 12 participants were involved in a mixed method research design which included completion of six tasks using three types of interfaces, Relevant In Context, Baseline and Focused (discussed in more detail in the next section), completion of satisfaction questionnaires and participation in semi-structured interviews. This research design permitted the collection of rich quantitative and qualitative data - e.g. a total of 72 tasks were performed (12 participants $\times 6$ tasks) and 1033 relevance judgments were analysed as part of this study. Actions were taken in order to counterbalance the effects of our small sample size, such as the use of a within-subjects design, as opposed to a between subjects one, and the performance of statistical tests where appropriate. Moreover, a final inspection of the data of this study suggested the presence of no extreme variations in performance 
Demian\&d Balatsoukas, Ms. No. CPENG-318 resubmitted to JCCE, September 2010

among participants, thus a decision was made to complete the study without recruiting more participants.

A set of six simulated tasks (Borlund, 2000) were developed. The purpose of the simulated tasks was to create a simple search scenario for participants in which they would need to assess the relevance of retrieved information. Traditionally, a simulated task presents information about a predetermined information need, including contextual information about how this information need originated and how the information obtained will be used. The selection of simulated tasks, over real information needs, serves the need of controllability over the type of data collected (i.e. a common basis for comparing data obtained across participants). However, as opposed to standard fixed tasks, simulated work tasks can lead to cognitively individualized interpretations of the information need, thus mimicking a more naturalistic relevance judgment process (i.e. each participant receives details related to the objectives of the search as well as the problem which has to be solved as opposed to a closed request for information). This is the reason why simulated tasks have been extensively used in mainstream IR experiments, such as TREC (Voorhees and Harnan, 2005) and INEX (Pharo, 2008). An example of the format of a simulated work task is presented in Figure 2.

The six tasks were equally divided into two groups of fact finding and decision making tasks. Each participant was asked to perform two tasks (one decision making and one fact finding) per interface ( 2 tasks $\times 3$ interfaces). Table 1 clarifies the difference between fact finding and decision making tasks.

A within-subjects design was employed that required all participants to perform the same set of tasks and interact with the three search result interfaces. At the 
Demian\&d Balatsoukas, Ms. No. CPENG-318 resubmitted to JCCE, September 2010

beginning of the user study each participant was instructed to complete the first task

(e.g. fact finding) and judge the relevance of the results presented in the first results page of the first interface (e.g. Baseline interface). During the evaluation of the results the participants were instructed to highlight with the mouse any terms or phrases that helped them to judge the relevance of the results (as suggested by Barry, 1994; Crystal and Greenberg, 2006) and assess them based on a 5-point scale: Too broad, Narrow, Exactly relevant, Partially relevant, Not relevant (see section on Granularity). After completing the first task in one interface participants were asked to continue with the second task (e.g. decision making) using the same interface condition. The same procedure was repeated for the remaining two interfaces, thus permitting the completion of a total of six tasks (both fact finding and decision making) using three interface conditions (Relevant In Context, Baseline and Focused). Participants' interaction with the search results was captured through the use of a screen recording software (Camtasia) with an audio recording function enabled in order to record their relevance judgments. Data collected included the time needed by participants to complete the tasks for each interface and the accuracy of their relevance judgments. Figure 3 presents a summary of the research design.

The sequence with which the interfaces and the tasks were presented to the participant was randomized to counterbalance the effects of learning. After performing both tasks using one interface and before proceeding to the evaluation of the next interface, participants were asked to fill in a satisfaction questionnaire. The satisfaction questionnaire used a 7-point scale to measure participants' overall satisfaction, confidence with the accuracy of their relevance judgments, how easy it was for participants to judge relevance and their satisfaction with the way the search results were presented to them. In addition, participants were asked to rate the 
Demian\&d Balatsoukas, Ms. No. CPENG-318 resubmitted to JCCE, September 2010

perceived level of difficulty of completing both types of tasks using that interface.

Finally, a semi-structured interview was conducted with each participant to provide a deeper evaluation of the interfaces (such as likes and dislikes regarding the presentation of search results) and cover any issues missed by the questionnaires.

\section{Prototype System: CoMem-XML}

In a previous study by Demian and Fruchter (2006b), three different prototypes, focused on one of the three aspects of ranking, granularity and context, were used allowing users to retrieve information from the same repository of engineering design content. The HitList focused on ranking and enabled users to enter keyword queries and retrieve results ranked by relevance in the same way as a web search engine. The Outline Tree focused on the granularity of the design content and allowed users to explore the repository by expanding sub-trees of the three-level hierarchy of content in the same way that most operating systems allow users to explore files and folders. The CoMem tool focused on context by allowing users visually to find a focal piece of information, identify items related to the focal information and finally, visualize those items in two dimensional Euclidean space.

A new prototype system, CoMem-XML places equal emphasis on ranking, granularity, and context. The following subsection describes the structure of the content in the CoMem-XML repository. The subsection after that describes the interface of the prototype in more detail, particularity how it incorporates ranking, granularity, and context. The final subsection describes two variants of CoMem-XML that were developed for a user study.

\section{The Collection}

The CoMem-XML content is hierarchical. A project object (e.g. Imaginary Hotel in 
Demian\&d Balatsoukas, Ms. No. CPENG-318 resubmitted to JCCE, September 2010

California) is made up of multiple discipline objects (e.g. the architectural layout of the hotel or the structural frame of the hotel). Each discipline object is made up of multiple components (a particular room or a particular structural column). Each component is further decomposed into two levels: Subcomponent (e.g. a joint at the end of a column) and Sub-subcomponent (e.g. a bolt in the joint) (Figure 4). This hierarchy is used to organize objects from the CAD models in the repository, but also (more importantly) to organize any textual or non-textual documents associated with construction projects: construction schedules, reports, journal papers, email archives, contracts, specifications.

The projects included in the CoMeM-XML system had a minimum of three levels of hierarchy (Project, Discipline, Building Component), a maximum of five levels (Project, Discipline, Building Component, SubComponent, Sub-subcomponent) and an average depth of 4.1 hierarchical levels per project. Each Project included several Disciplines and Building Components coded and stored in XML files. The relatively small number of nodes at the lower levels meant that the average branching factor of the hierarchy was just over 2. Each level of a project's hierarchy (Discipline or Component) included metadata that described its content, such as name, cost, design data and maintenance data. These metadata were encoded using AEC-XML (Figure 5). AEC-XML is an XML Schema used to create data for construction projects and building modeling. It uses the Industry Foundation Classes (IFC) data model. Finally, the total number of XML nodes in the collection, across all the hierarchical levels, was approximately 2.500 . These came from 17 projects, 72 disciplines, 170 building components, 224 SubComponents and 121 Subsubcomponents. 
Demian\&d Balatsoukas, Ms. No. CPENG-318 resubmitted to JCCE, September 2010

\section{Relevance ranking, Granularity and Context in CoMem-XML}

The CoMem-XML system uses the vector space model (Salton et al., 1975) in order to index and retrieve the information included in the hierarchical XML files following a method suggested by Carmel et al (2003). The three classic information retrieval models are the Boolean model, the vector model, and the probabilistic model (BaezaYates and Ribeiro-Neto 1999, Chap. 2 gives an overview). It is widely recognized that the Boolean model (which involves binary keyword matching) is too limiting because of its inability to recognize partial matches. There is ongoing discussion as to which of the two remaining models outperforms the other. CoMem-XML adopts the vector model because of its relative simplicity. Since the present study is focused on the user interface and presentation of search results, computational issues of retrieval and ranking are considered beyond the scope of this paper. A particular characteristic of the retrieval process, however, was the calculation of weights for both the content in the CoMem-XML system, as well as the XML metadata elements that defined the context and structure of that content. For example, <legislation $>C$ $108 / 01</$ legislation $>$ was an indexing unit that represented the content $(C-108 / 01)$ and defined its meaning, that $C-108 / 01$ is a piece of legislation. Another example, is the fragment <building_component>First floor kitchen</building_component> that defined the structural level of the content (i.e. that the first floor kitchen is a Building Component and therefore, part of a particular Discipline and Project). Queries were performed using NEXI. NEXI is a query language developed at INEX which provides the opportunity to search a collection of XML documents using both content (full text) as well as structural elements (similar to Xquery and Xpath technologies) (Manning et al., 2008).

After a query was executed, the results were displayed as a ranked list of items grouped by Project. Projects themselves were ranked by relevance. The calculation 
Demian\&d Balatsoukas, Ms. No. CPENG-318 resubmitted to JCCE, September 2010

of the relevance score of a Project was taken as the average of the individual relevance scores of the constituent items of that Project (i.e. Discipline, Building Component, Sub-component and Sub-subcomponent). When a set of siblings were retrieved, those were ranked by relevance and grouped under their parent. For example, if relevant information was identified within a Building Component, then information about the Discipline within which the Building Component occurred was displayed as well (Figure 6). In addition, the user had the option of exploring other Building Components and Sub-components within that Discipline item, regardless of their relevance to the user's query. This was facilitated through the expand link which appears next to each ancestor of the retrieved item. The purpose of the expand function was to display further associative items (Nielsen, 1999) that occurred at the same hierarchical level as the retrieved relevant item. The search result interface therefore provides as much contextual information as possible for users to explore the context of a retrieved information item and decide upon its relevance to their task at hand. Finally, the user had the opportunity to open a new window with the actual content contained at that node.

In this search result interface, granularity can be defined as the position of a retrieved item within the hierarchy; a Project which might refer to a whole building is clearly a coarser grain of content than a Sub-subcomponent which might refer to a tiny building constituent. Context refers to an item's relationships with other items, particularly its ancestors, descendents and siblings. Context can be conceptualized by positioning any granular item in the two dimensional space of breadth against depth. Depth refers to direct ancestors or descendents of an item (e.g. for a Building Component, that would be its parent Discipline and grandparent Project, or its children Subcomponents and grandchildren Sub-subcomponents). Breadth represents contextual items from the same level of the hierarchy (e.g. for a Building 
Demian\&d Balatsoukas, Ms. No. CPENG-318 resubmitted to JCCE, September 2010

Component, that would be any other related Building Components in the repository, whether in the same project or from other projects) (Figure 7).

XML makes use of both depth and breadth in the description of content, and search result interfaces can take advantage of this two dimensional space in order to present the retrieved results in context. This approach is appropriate for more structured engineering content and differs from traditional IR applications (such as web search engines and bibliographic systems) where an emphasis is placed on the retrieval and presentation of whole documents as opposed to the various components of which a document consists. Moreover, the search result interface of CoMem-XML does not present a hierarchical list of all the contents of a specific project (as in the case of a table of contents or a static tree-based structure, such as the Outline Tree or Windows File Explorer) but filters to show only the relevant hierarchical components and displays them both in context and ranked order. In this manner, both ranking and context are communicated within a single search result interface. This is of particular relevance to the information searching processes of designers and engineers where the item of enquiry should be both relevant and indicative of its position in the parts hierarchy (Demian and Fruchter, 2006b; Hertzum and Pejtersen, 2000). In this case understanding and exploring the context of the retrieved item is important for engineers to take decisions about it's potential relevance.

In order to examine and evaluate the CoMem-XML system and the underlying hypothesised importance of granularity and context, two further search result interfaces were developed. These represented granularity and context in different ways in the search result interface. A more detailed description of these interfaces and the research design of the user study are discussed in the next subsection. 
Demian\&d Balatsoukas, Ms. No. CPENG-318 resubmitted to JCCE, September 2010

\section{Focused and Baseline Versions of Prototype}

In the previous subsection a search result interface was presented that ranks

Projects by relevance. Within each Project all relevant items (Discipline and Building

Component objects) of the particular Project are displayed and sorted by relevance

as well. Finally, each item was accompanied by its context at various granularity

levels (i.e. related Discipline, Building Component and Sub-component objects). For the purpose of clarity this type of interface will be called Relevant in Context $(R I C)$

(Figure 6).

In addition, two further search result interfaces were developed to test the importance of granularity and context. These were: the Focused interface, and the Baseline interface. In the case of the Focused interface the search results present only a ranked list of the most relevant items, regardless of granularity or project grouping. However, the retrieved items appear out of context, i.e. outside the project context, and they are ranked by relevance (Figures 8 and 9). Again, for each retrieved item, links were provided to display the content of that item and to list all its children in the hierarchy.

Finally, the Baseline interface lists only the project titles ranked by relevance (along with some metadata, such as ID number and date of last modification). Users of the Baseline interface had the opportunity to explore all the components of each project. However, individual items were not sorted by relevance (as in the case of the Relevant in Context interface) but retained their original position in the hierarchy. This interface (Figures 10 and 11) was similar to a static tree-based hierarchical structure, such as the Windows File Explorer or the Outline Tree viewer. 
Demian\&d Balatsoukas, Ms. No. CPENG-318 resubmitted to JCCE, September 2010

In order to isolate any other effects of interface design on user interaction, the three

search result interfaces did not differ in terms of typographical characteristics (such as font type and size and background colour). In addition, the same method for calculating relevance was used across the three interfaces. Finally, the three interface versions were displayed to participants in the study using Internet Explorer v7.0 on a 14-inch laptop screen.

\section{Results}

\section{Demographic data}

Twelve students and researchers of the Department of Civil and Building Engineering at Loughborough University participated in the study. Three participants were undergraduate students, five were postgraduate research students and the remaining four were research staff. All participants were experienced users of the WWW. In terms of WWW use, eight participants indicated that they used the WWW for more than 10 hours per week and four participants between five to 10 hours per week. Participants reported using the WWW for a variety of reasons including academic research, entertainment, communicating with peers and purchasing goods and services. They were also frequent users of various Enterprise Information Systems such as the library Online Public Access Catalogue, scholarly databases, web search engines and corporate databases. Finally, all participants reported having extensive professional experience and indicated that they had previously used a corporate database to find information about construction management projects either as part of an internship or during a professional career in the construction industry. (The Department is renowned for its close links with industry and the industrial experience of its academic staff and researchers; even undergraduate students predominantly take "sandwich courses" where they spend a year in industry in the middle of their academic studies as part of their undergraduate degree programme.) 
Demian\&d Balatsoukas, Ms. No. CPENG-318 resubmitted to JCCE, September 2010

\section{Time spent in the evaluation of search results}

Test participants were asked to indicate when they had completed the task. In this way, the time taken to evaluate the search results was measured. Participants performed both tasks faster using the RIC interface followed by the Focused interface and finally the Baseline interface. Figure 12 shows the differences between the three interfaces across the two sets of tasks (fact finding and decision making). In particular, the difference between the $R I C$ and the other two interfaces was more pronounced for decision making tasks than for focused tasks. The Focused interface was the least time efficient interface for the decision making tasks but performed better than the Baseline interface for the fact finding tasks. A two-way within subjects ANOVA test was performed in order to investigate whether there were any significant differences between the three interfaces and the tasks performed. The ANOVA test showed that the three interfaces differed significantly at the 0.05 level. The Bonferroni pairwise comparisons showed the difference observed between the RIC and the other two interfaces was significant for both types of tasks. However, the differences recorded between the Baseline and the Focused interfaces were not found to be statistically significant.

\section{Accuracy of relevance judgments}

During the user study, participants were asked to assess the relevance of the results retrieved by the system. These participants' relevance judgments were compared to those made by two experts before the user study. The results show that participants made more accurate relevance judgments using the $R I C$ interface $(65 \%)$, than the Baseline (54\%) and Focused (43\%) interfaces (Table 2). A more in depth analysis across the two tasks shows that the $R I C$ interface enabled more accurate relevance judgements to be made than the other two interfaces for the decision making task (Figure 13). The results of a two-way within subjects ANOVA test show that this 
Demian\&d Balatsoukas, Ms. No. CPENG-318 resubmitted to JCCE, September 2010

difference is statistically significant at the 0.05 level. However, no significant differences were observed between the three interfaces in the case of the fact finding task. The differences observed between the two types of tasks were significant, with the level of accuracy being higher for fact finding than for decision making tasks. This was evident in the case of the Baseline and Focused interfaces where participants made less accurate relevance judgments for the decision making task.

\section{User satisfaction}

Analysis of the satisfaction questionnaire shows that participants were more satisfied with the RIC interface than the Baseline or the Focused interfaces (Table 3). This was evident across all the satisfaction variables used in the questionnaire, such as satisfaction, stimulation, ease of use of the interface, confidence about the relevance judgments, ease of judging relevance and satisfaction with the presentation of the results.

A set of Friedman tests were performed in order to test the significance of the differences between the three interfaces. The results show that satisfaction, confidence with the relevance judgments and satisfaction with the presentation of search results varied significantly across the three interfaces at the 0.01 level. In addition, significant differences were observed in the case of the perceived difficulty in judging relevance across the three interfaces at the 0.05 level. Based on these findings a set of Wilcoxon tests were conducted in order to ascertain where these differences occurred. In this case the tests compared multiple pairs of interfaces (i.e., Baseline and Focused, Baseline and RIC, Focused and RIC) for each variable. The results show that the differences observed between the $R I C$ and the other two interfaces were significant for all variables. However, no statistically significant 
Demian\&d Balatsoukas, Ms. No. CPENG-318 resubmitted to JCCE, September 2010

differences were observed between the Focused and Baseline interface. In the latter case, an exception holds for the variable that examined satisfaction with the presentation of search results. Based on the Wilcoxon tests significant differences (at the 0.05 level, two-tailed) were observed between the Baseline and the Focused interface with the former scoring higher than the latter.

The satisfaction questionnaire also asked participants to indicate the perceived difficulty of completing the two tasks (fact finding and decision making) using the three interfaces (Table 4). The Likert scores show that participants found it easier to perform both types of tasks using the RIC interface. The Focused interface was the most difficult for participants to perform both tasks. It is noteworthy that participants found it more difficult to perform the decision making task than the fact finding task for all three interfaces. The Friedman tests show that these differences were significant across the three interfaces at the 0.01 level for both types of tasks.

\section{User feedback (interviews)}

To obtain more detailed feedback, each participant was interviewed after using all three interfaces to perform both types of tasks. Overall, the majority of participants in the study (ten of the twelve) preferred the presentation of results in the Relevant in Context interface. The main advantage of this interface over the Focused and the Baseline versions was that it displayed only the items that were relevant to the task at hand. By eliminating irrelevant items, participants were able to focus on a smaller subset of relevant project hierarchies that did not take up a lot of space on the screen. This was more conducive to evaluating the items of a project, and reduced the need to scroll down long lists of irrelevant components. Participants also expressed preference for the fact that if multiple siblings in the hierarchy were retrieved, those were ranked by relevance. 
Demian\&d Balatsoukas, Ms. No. CPENG-318 resubmitted to JCCE, September 2010

Another characteristic of the Relevant in Context interface was the ability to explore the context within which retrieved relevant information occurred. For example, when a relevant item was retrieved at the Subcomponent level, participants were able to explore the Building Component, Discipline and Project within which that item occurred. In addition, participants were able to browse downwards the hierarchy and list the Sub-subcomponents included within that Subcomponent or even explore what other Sub-component items were available at the same level of granularity. Participants found this feature particularly useful because it enabled them to judge relevance according to the place or position of the relevant information within the project structure. In this manner, granularity was a useful means for understanding information. This function was not available in the case of the Focused interface that displayed retrieved items out of context. With the Focused interface, participants reported difficulty in judging the relevance of the retrieved information when it was displayed in isolation, out of context.

Two participants preferred the Baseline interface for judging relevance, arguing that they liked the presentation of all the items of a project even though this meant that some of them were not relevant to the task at hand. Participants explained that the task of deciding which items were relevant should be left to them as human users, and not automated by the system's retrieval mechanism. This feeling, however, can be explained if those participants preferred browsing rather than querying the contents of the repository. Finally, none of the participants in the study expressed a preference for the presentation of results in the Focused interface. This interface made it difficult for them to understand the context of the retrieved information. In a few cases, participants indicated that the information retrieved was meaningless. 
Demian\&d Balatsoukas, Ms. No. CPENG-318 resubmitted to JCCE, September 2010

During the interviews, participants proposed possible improvements to CoMem-XML.

A function which was widely requested was the ability to view which items from different projects are relevant to any given item. If a relevant item within a project hierarchy was found the system should also present related items from other projects. More superficially, participants requested the inclusion of visual or textual hints in order to denote the type of information included under each level of the hierarchy (such as a CAD file, PDF document or a spreadsheet). Although the format of the information was indicated in the in the details view, after clicking on a specific item listed on the search result interface, some participants suggested that information about the format should be visible in the search result interface itself.

\section{Discussion}

When retrieving from repositories of content about construction projects, participants needed less time to judge relevance and made more accurate relevance judgments using the RIC interface than the Focused and Baseline interfaces. This was more pronounced for decision making tasks that involved a more complex relevance judgment process than the simpler fact finding tasks. The performance results are aligned with the user satisfaction results. Participants were more satisfied, felt more confident, and found it easier to judge relevance using the $R I C$ interface. These effects can be ascribed to the contextual information provided by the $R I C$ interface for each retrieved item, and the fact that it includes only relevant information in the search results.

These findings support the results of other studies that investigated the information searching behaviour of engineers (Kraaijenbrick, 2007; Demian and Fruchter, 2006b). For example, in the present study, the Focused interface, which did not display information about the context of retrieved items, was the least time efficient 
Demian\&d Balatsoukas, Ms. No. CPENG-318 resubmitted to JCCE, September 2010

and the most error prone interface (in terms of the accuracy of relevance judgments).

Participants in the interviews found it difficult to understand the context of the information displayed in this interface and to judge its relevance. Although the Focused interface provided the option of exploring all the contents of a project in a different window, this feature was only used by few participants. Similar were the findings reported by Kraaijenbrick (2007) who found that participants expected to browse the depth and the breadth of the information in order to understand its context. This feature, however, was evident in the case of the RIC interface. Similar results are reported in the study of web search engines. Vegas et al (2007) found that users were more satisfied with search result interfaces that visualise the context of the retrieved web-pages through the use of the DocBall metaphor. Therefore, it appears that context, in the form of an item's position in the hierarchy, can increase the value of the information scent (Pirolli, 2007) communicated to users during the relevance judgment process. This happens because relevant information that is presented at lower levels of granularity (such as Sub-component and Subsubcomponent) can be contextualised and clarified by the parent nodes, such as Building Component, Discipline and Project. In a previous study, Pirolli et al (2003) found that the use of semantics that appropriately described the contents of parent nodes helped participants to predict the place of child nodes in a hierarchy more accurately.

Demian and Fruchter (2006b) found that context was more critical in the case of complex and exploratory tasks. The findings of the present study concur that context was more important for the completion of complex tasks, such as decision making tasks, rather than fact finding tasks. For example, although the differences between the $R I C$ and the other two interfaces were significant for decision making tasks, they were not statistically significant for fact finding tasks. This can be explained by the 
Demian\&d Balatsoukas, Ms. No. CPENG-318 resubmitted to JCCE, September 2010

fact that a response to a simple fact finding task could be obtained from small chunks of relevant information with narrow context. However, the decision making tasks required a deeper understanding of the context of each component.

Context was not the only factor that influenced user performance and satisfaction during the relevance judgment process. Relevance ranking and filtering is equally important in search result interfaces that present information at various levels of granularity. Although both the Baseline and the $R I C$ interfaces displayed information in context, users of the latter performed better than users of the former. This can be explained by the fact that the $R I C$ interface displayed only relevant project components, as opposed to the Baseline interface that included all contents (both relevant and irrelevant). The presentation of only relevant information reduced the need for scrolling and increased the amount of relevant information that was displayed within the area of visual attention of the user (Pirolli 2007; Duchowski 2007).

Finally, the present study extends that conducted by Demian and Fruchter (2006b) by proposing that query-driven textual IR systems should leverage on both context (presentation of results at different levels of granularity) and relevance (ranking by relevance) to optimise engineers' performance and satisfaction when judging relevance.

\section{Conclusions: Granularity and context in BIM and beyond}

The purpose of this study was to investigate the effects of search result interfaces (particularly the aspects of granularity and context) in systems for searching archives of construction documents. A user study was conducted to compare three prototypes. The findings showed that participants performed better and were more 
Demian\&d Balatsoukas, Ms. No. CPENG-318 resubmitted to JCCE, September 2010

satisfied with the RIC interface because it presented only relevant information in context and with careful attention to granularity (grouping items by level of granularity and facilitating the ordered navigation of the parts hierarchy).

Although caution must be exercised when generalising these findings, the contributions of this research are subtle but not trivial. From a theoretical point of view, the findings of this study advance our understanding of the information seeking behaviour of civil engineers, and especially their relevance judgment in query-driven repositories of construction content. To date, studies were focused on the importance of granularity and context in 2D and 3D visual interfaces. However, this research examined the relevance judgment behaviour of engineers in the case of textual and query-driven search result interfaces and showed how different textual interfaces can accommodate granularity and context, but also what are their effects on the relevance judgment behaviour. Since there is still a debate among researchers about the effects of visual and textual interfaces on users' cognitive load and relevance judgment behaviour, it is important to gain a deeper understanding of users' interaction. Therefore, the theoretical contribution of this research is a step towards this end. From a methodological point of view, this research suggested a novel research design for the user-centred evaluation of XML based repositories of construction content. This involved a combination of techniques used in mainstream information science research (such as the use of simulated work tasks and the recording of individual relevance judgments based on five relevance categories appropriate for the evaluation of granularity and context) and usability testing (such as satisfaction questionnaires and debriefing semi-structured interviews). Finally, the practical contributions of this research involve the development of recommendations for the design of search result interfaces for structured query-driven repositories of construction documents. More specifically, it is suggested that query-based retrieval 
Demian\&d Balatsoukas, Ms. No. CPENG-318 resubmitted to JCCE, September 2010

from such repositories should rely on the relevance of individual items, but must also leverage links between retrieved items and their relatives in the repository. Retrieved items can be ranked by relevance, but links in the search result interface should be provided to the ancestors, descendants and siblings of each item. Where appropriate, listings of those sets of relatives can also be grouped by level of granularity and ranked by relevance to the original query. Such functionality would enable users to complete retrieval tasks more rapidly and make more accurate relevance judgements. These effects are particularly pronounced for more complex decision making tasks.

Moreover, the findings of this study still have important implications for the design of BIM systems. As the scope of BIM expands, it is becoming impossible for a single model to include all conceivable information. Some documents and information must remain external to the BIM platform. The BIM could still play an important role in managing this external content. Linking external documents to the BIM would enable the relationships between elements within the BIM to be exploited to manage external documents. Such systems will need careful management of granularity and context and would therefore be informed by this research. The present study paves the way for future research into the use of a BIM as a visual index for project documents.

\section{Acknowledgements}

This research was supported by the Leverhulme Trust through a Fellowship to Peter Demian. 
Demian\&d Balatsoukas, Ms. No. CPENG-318 resubmitted to JCCE, September 2010

\section{References}

Agdas. D. and Ellis. R.D., (2010). "The potential of XML technology as an answer to the data interchange problems of the construction industry." Construction Management and Economics (July 2010), 28, pp, 737-746.

Allen, T. (1977). Managing the flow of technology. MIT Press, Cambridge, MA.

Anumba, Cm.J., Egbu. C.O., Carrillo. P.M. (eds.) (2005). Knowledge Management in Construction, Blackwell Publishing. 2005.

Austin, S., Baldwin, A., Baizhan, L. and Waskett, P. (2000). "Analytical design planning technique (ADePT): a dependency structure matrix tool to schedule the building design process". Construction Management and Economics (2000) 18, 173-182.

Baeza-Yates, R., and Ribeiro-Neto, B. (1999). Modern information retrieval, Addison-Wesley, Harlow, U.K.

Barry, C. (1994). “User-defined relevance criteria: an exploratory study.” Journal of the American Society for Information Science and Technology, 45(3), 149159.

Betsi, S., Lalmas, M., Tombros, A., and Tsikrika, T. (2006). “User expectations from XML Element retrieval." In E.N. Efthimiadis, S.T. Dumais, D. Hawking, \& K. Järvelin (eds.), Proceedings of the 29th Annual International ACM SIGIR Conference on Research and Development in Information Retrieval (pp. 611612). NewYork: ACM Press.

Borlund, P. (2000). "Experimental components for the evaluation of interactive information retrieval systems", Journal of Documentation, 56(1) $71-90$.

Brilakis, I.K., and Soibelman, L., (2008). "Shape-Based Retrieval of Construction Site Photographs." ASCE Journal of Computing in Civil Engineering. 22, 14 (2008). 
Demian\&d Balatsoukas, Ms. No. CPENG-318 resubmitted to JCCE, September 2010

Caldas C., Soibelman L. and Han, J. (2002). "Automated Classification of Construction Project Documents." ASCE Journal of Computing in Civil Engineering, 16(4) 234-243.

Carmel, D., Maarek, Y., Mandelbrod, M., Mass, Y., and Soffer, A. (2003). "Searching XML documents via XML fragments." In Proceedings of the 26th Annual International ACM SIGIR Conference on Research and Development in Information Retrieval (pp. 151-158). New York: ACM Press.

Chakrabarti, A., Feineman, S., and Fuentevilla, W. (1983). "Characteristics of sources, channels and contents for scientific and technical information systems in industrial $R$ an D. IEEE Transactions on Engineering Management, EM-30(2), pp. 83-88.

Crystal, A., and Greenberg, J. (2006). "Relevance criteria identified by health information users during Web searches." Journal of the American Society for Information Science and Technology, 57(10), 1368-1382.

Demian, P., and Fruchter, R. (2005). "Measuring relevance in support of design reuse from archives of building product models." ASCE Journal of Computing in Civil Engineering, 19(2), 119-136.

Demian, P., and Fruchter, R. (2006a). "An ethnographic study of design knowledge reuse in the architecture, engineering and construction industry." Research in Engineering Design, 16, 184-195.

Demian, P., amd Fruchter, R. (2006b). "Methodology for usability evaluation of corporate memory design reuse systems." Journal of Computing in Civil Engineering, 20(6) 377-389.

Duchowski, A. (2007). Eye tracking methodology: theory and practice. SpringerVerlag. 
Demian\&d Balatsoukas, Ms. No. CPENG-318 resubmitted to JCCE, September 2010

Eastman, C., Teicholz, P., Sacks R, and Liston K, (2008). BIM Handbook: A Guide to Building Information Modeling for Owners, Managers, Designers, Engineers and Contractors. John Wiley \& Sons.

Eastman, C., (1999). Building product models: computer environments supporting design and construction. CRC Press, Inc. Boca Raton, FL, USA

Edmunds, A. and Morris, A., (2000). "The problem of information overload in business organisations: a review of the literature." Int. J. of Information Management , 20(1) 17-28.

Ellis, D. and Haugan, M. (1997). "Modelling the information seeking patterns of engineers and research scientists in an industrial environment." Journal of Documentation, 53(4) 384-403.

Fidel, R. and Green, M (2004). “The many faces of accessibility: engineers' perception of information sources." Information Processing and Management, 40(3) 563-581.

Gerstberger, P., and Allen, T. (1968). "Criteria used by development engineers in the selection of an information source." Journal of Applied Psychology, 52(4), 272-279.

Gray, C. and Hughes, W. (2001). "Building Design Management." ButterworthHeinemann.

Hammer-Aebi, B., Christensen, K., Lund, H., and Larsen, B. (2006). “Users, structured documents and overlap: Interactive searching of elements and the influence of context on search behaviour." In I. Ruthven, P. Borlund, P. Ingwersen, N. Belkin, A. Tombro, \& P. Vakkari (eds.), Proceedings of the First Symposium on Information Interaction in Context (IliX 2006) (pp. 80-94). Copenhagen, Denmark: Royal School of Library and Information Science. 
Demian\&d Balatsoukas, Ms. No. CPENG-318 resubmitted to JCCE, September 2010

Hertzum, M., and Pejtersen, A. (2000). "The information seeking practices of engineers: Searching for documents as well as for people." Information Processing \& Management, 36(5), 761-778.

Hicks, B .J., Dong, A., Palmer, R., Mcalpine, H.C., (2008). "Organizing and managing personal electronic files: A mechanical engineer's perspective." ACM Transactions on Information Systems, 26(4) (September 2008).

Howell, I. and Batcheler, B. (2010). "BIM could be better: Ask for links," July 27, 2010, BIM Channel, retrieved from http://www.bimchannel.com/articlebim_could_be_better_ask_for_links-11.html (26 August 2010).

INEX. (2009). "Initiative for the evaluation of XML retrieval." Retrieved 7 May 2010, from http://inex.is.informatik.uni-duisburg.de/

KIM (2006). "KIM Grand Challenge Project." Retrieved from http://wwwedc.eng.cam.ac.uk/kim/ on 7 May 2010.

Kiziltas, S. and Akinci, B., (2010). "Automated Generation of Customized Field Data Collection Templates to Support Information Needs of Cost Estimators." Journal of Computing in Civil Engineering. 24(2) 129-139.

Koo, B. and Fischer, M. (2000). "Feasibility Study of 4D CAD in Commercial Construction." Journal of Construction Engineering and Management, 126(4) 251-260.

Kovacevic M, Jian-Yun, N., and Davidson, C., (2008). "Providing Answers to Questions from Automatically Collected Web Pages for Intelligent Decision Making in the Construction Sector.", Journal of Computing in Civil Engineering. 22(1) 3-13.

Kraaijenbrick, J. (2007). "Engineers and the Web: An analysis of real life gaps in information usage." Information Processing \& Management, 43(5), 13681382. 
Demian\&d Balatsoukas, Ms. No. CPENG-318 resubmitted to JCCE, September 2010

Kunz, J.C., Christiansen, T.R., Cohen, G.P., Jin, Y., and Levitt, R.E. (1998). "The virtual design team". Communications of the ACM, 41(11), 84-91.

Kwasitsu, L. (2003). "Information-seeking behaviour of design, process and manufacturing engineers." Library and Information Science Research, 25(4), 459-476.

Larsen, B., Tombros, A., and Malik, S. (2006). "Is XML retrieval meaningful to users? Searcher preferences for full documents vs. elements." In Proceedings of the 29th Annual International ACM SIGIR Conference on Research and Development in Information Retrieval (pp. 663-664). NewYork: ACM Press.

Leckie, G., Pettigrew, K., and Sylvain, C (1996). "Modeling the information seeking of professionals: a general model derived from research on engineers, health care professionals and lawyers." Library quarterly, vol. 66(2), pp. 161-193.

Lehtonen, M. (2006). "Designing user studies for XML retrieval." In Proceedings of the 29th Annual International ACM SIGIR Conference on Research and Development in Information Retrieval (pp. 28-34). NewYork: ACM Press

Lin, K. and Soibelman, L. (2009). “Incorporating Domain Knowledge and Information Retrieval Techniques to Develop an Architectural/Engineering/Construction Online Product Search Engine.” Journal of Computing in Civil Engineering. 23(4), 201-210.

Linkedln, 2010. Discussion Topic: "You can link external data to BIM objects..." in the Linkedln BuildingSMART (unofficial) group.

Lyman, P. and Varian, H.R. (2003). "How Much Information.". Retrieved from http://www.sims.berkeley.edu/how-much-info-2003 on 20 May 2010.

Malik, S., Tombros, A., and Larsen, B. (2006). "The interactive track at INEX." ACM SIGIR Forum, 41(1), 67-74.

Manning, C., Raghavan, P., and Schutze, H. (2008). Introduction to information retrieval. NewYork: Cambridge University Press. 
Demian\&d Balatsoukas, Ms. No. CPENG-318 resubmitted to JCCE, September 2010

Nielsen, J. (1999). "User interface directions for the Web." Communications of the ACM, 42(1), 65-72.

Pehcevski, J. (2006). "Relevance in XML retrieval: The user perspective." In Proceedings of the 29th Annual International ACM SIGIR Conference on Research and Development in Information Retrieval (pp. 35-42). NewYork: ACM Press.

Pharo, N. (2008). "The effect of granularity and order in XML element retrieval." Information Processing \& Management, 44(5), 1732-1740.

Pinelli, T., Bishop, A., Barclay, R. and Kennedy, J. (1993). "The information seeking behavior of engineers." In Kent, A. and Hall, C. (eds.), Encyclopaeia of library and information science, 52, 167-201. New York: Marcel Dekker.

Pirolli, P. (2007). Information foraging theory: adaptive interaction with information. Oxford: Oxford University press.

Pirolli, P., Card, S., and Wege, M. (2003). "The effects of information scent on visual search in the Hyperbolic Tree browser." ACM Transactions on ComputerHuman Interaction, 10(1), 20-53.

Rezgui, Y. (2001). "Review of information and the state of the art of knowledge management practices in the construction industry." The Knowledge Engineering Review, 16(3) 241-254. Cambridge University Press.

Rezgui Y, 2006. "Ontology-Centered Knowledge Management Using Information Retrieval Techniques." Journal of Computing in Civil Engineering. 20(4), 261270.

Rubin, J., and Chisnell, D. (2008). Handbook of usability testing. NewYork: Wiley. Salton, G., Yang, C., and Yu, C. (1975). "A theory of term importance in automatic text analysis." Journal of the American Society for Information Science, 26(1) $33-44$. 
Demian\&d Balatsoukas, Ms. No. CPENG-318 resubmitted to JCCE, September 2010

Shen, G., Brandon, P., and Baldwin. A. (eds.), (2009). Collaborative Construction Information Management. Spon Press.

Shen, Z. and Issa, R.R.A.(2010). "Quantitative evaluation of the BIM-assisted construction detailed cost estimates." ITcon, 15, 234-257.

Tan, H.C., Anumba, C.J., Carrillo, P.M., Bouchlaghem, D., Kamara, J., and Udeaja, C. (2010). Capture and Reuse of Project Knowledge in Construction. John Wiley and Sons, 2010.

Vegas, J., Crestani, F., and de la Fuente, P. (2007). "Context representation for Web search results." Journal of Information Science, 33(1), 77-94.

Voorhees, E. and Harman, D. (2005). Experiment and evaluation in information retrieval. MIT Press.

Ye, Y., and Fischer, G. (2002). "Supporting reuse by delivering task-relevant and personalised information." In Proceedings of the 24th International Conference on Software Engineering, pp. 513-523.

Wachsmuth, I., and Meyer-Fujara, J (1990). "Addressing the retrieval problem in large knowledge bases." In Proceedings of the $3^{\text {rd }}$ Conference in Computational intelligence (Cl-90), Milano, University of Bielefeld (MOSYSReport 3), pp.1-13.

Wu, C. T. (2005). An introduction to object-oriented programming with Java. 4th ed. New York: McGraw-Hill Higher Education. 
Demian\&d Balatsoukas, Ms. No. CPENG-318 resubmitted to JCCE, September 2010

\begin{tabular}{|c|c|}
\hline $\begin{array}{l}\text { Fact finding } \\
\text { (the objective is to find } \\
\text { a specific piece of } \\
\text { information) }\end{array}$ & $\begin{array}{l}\text { Please find information about the renovation of historical } \\
\text { buildings }\end{array}$ \\
\hline $\begin{array}{l}\text { Decision making } \\
\text { (the objective is to } \\
\text { select the best option } \\
\text { among alternatives) }\end{array}$ & $\begin{array}{l}\text { You need to develop an action plan for the renovation of } \\
\text { historical buildings in the area of Leicestershire. You have } \\
\text { been given a budget of } £ 3000.000 \text {. The renovation plan } \\
\text { should also involve the installation of green technologies } \\
\text { for lowering the carbon footprint of the building. Which of } \\
\text { the past projects plans that dealt with the renovation of } \\
\text { historical buildings should be considered for reuse in the } \\
\text { present situation? }\end{array}$ \\
\hline
\end{tabular}

Table 1: Examples of fact finding and decision making tasks 
Demian\&d Balatsoukas, Ms. No. CPENG-318 resubmitted to JCCE, September 2010

\begin{tabular}{lccc} 
& Baseline & Focused & RIC \\
\hline $\begin{array}{l}\text { Number of relevance } \\
\text { judgments }\end{array}$ & 498 & 167 & 368 \\
\hline Number of correct relevance & 270 & 72 & 242 \\
Judgments & & \\
\hline & & & \\
Percentage (\%) of correct & 54 & 43 & \\
relevance judgments & & \\
\hline 2. Accuracy of relevance judgments. & & \\
\end{tabular}

Table 2. Accuracy of relevance judgments. 
Demian\&d Balatsoukas, Ms. No. CPENG-318 resubmitted to JCCE, September 2010

\begin{tabular}{lccc} 
& Baseline & Focused & RIC \\
\hline Not satisfied - Very satisfied & 3.3 & 3.8 & 5.4 \\
\hline Dull - Stimulating & 3.5 & 3.5 & 3.7 \\
\hline Hard to use - Easy to use & 4.5 & 5.2 & 5.4 \\
\hline Not at all confident -Confident with & 3.3 & 3.6 & 4.2 \\
relevance judgment & & & \\
\hline Not at all easy - Easy to judge & 3.3 & 3 & 4.2 \\
relevance & & & \\
\hline Did not like - Liked the & & & \\
presentation of results & 3.8 & 2.9 & \\
\hline Table 3. User satisfaction with the interfaces. Likert scores, 7-point scale
\end{tabular}


Demian\&d Balatsoukas, Ms. No. CPENG-318 resubmitted to JCCE, September 2010

Type of task

Baseline Focused RIC

The completion of task $\mathrm{A}$ (fact finding task)

was difficult:

3.8

5.2

3.3

Strongly disagree (1) - Strongly agree (7)

The completion of task B (decision

making) was difficult:

4.8

5.3

3.8

Strongly disagree (1) - Strongly agree (7)

Table 4. Difficulty in completing the two tasks. Likert score, 7-point 


\section{Document B}

\section{Element}

Element

Element

Document A

\section{Element \\ Element}

Document $\mathrm{C}$

Element

Element

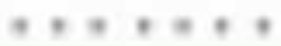




\section{Simulated situation (task code): Fact finding 1}

\section{Simulated work task situation:}

I am a contractor working on a project to renovate a listed building. I need to find any relevant documents/information about renovation of listed buildings. Buildings are listed by the UK government when they have special historical or architectural value and therefore need to be protected by law. Most of the relevant documents would not be related to any particular projects, but would be general legal documents and guidelines issued by the Government. Some documents might be from past renovation projects that I or my company has worked on: e.g. drawings, contracts, specifications from previous projects.

Indicative request (search keywords):

Find information about the renovation of historical buildings 
Click here to download high resolution image

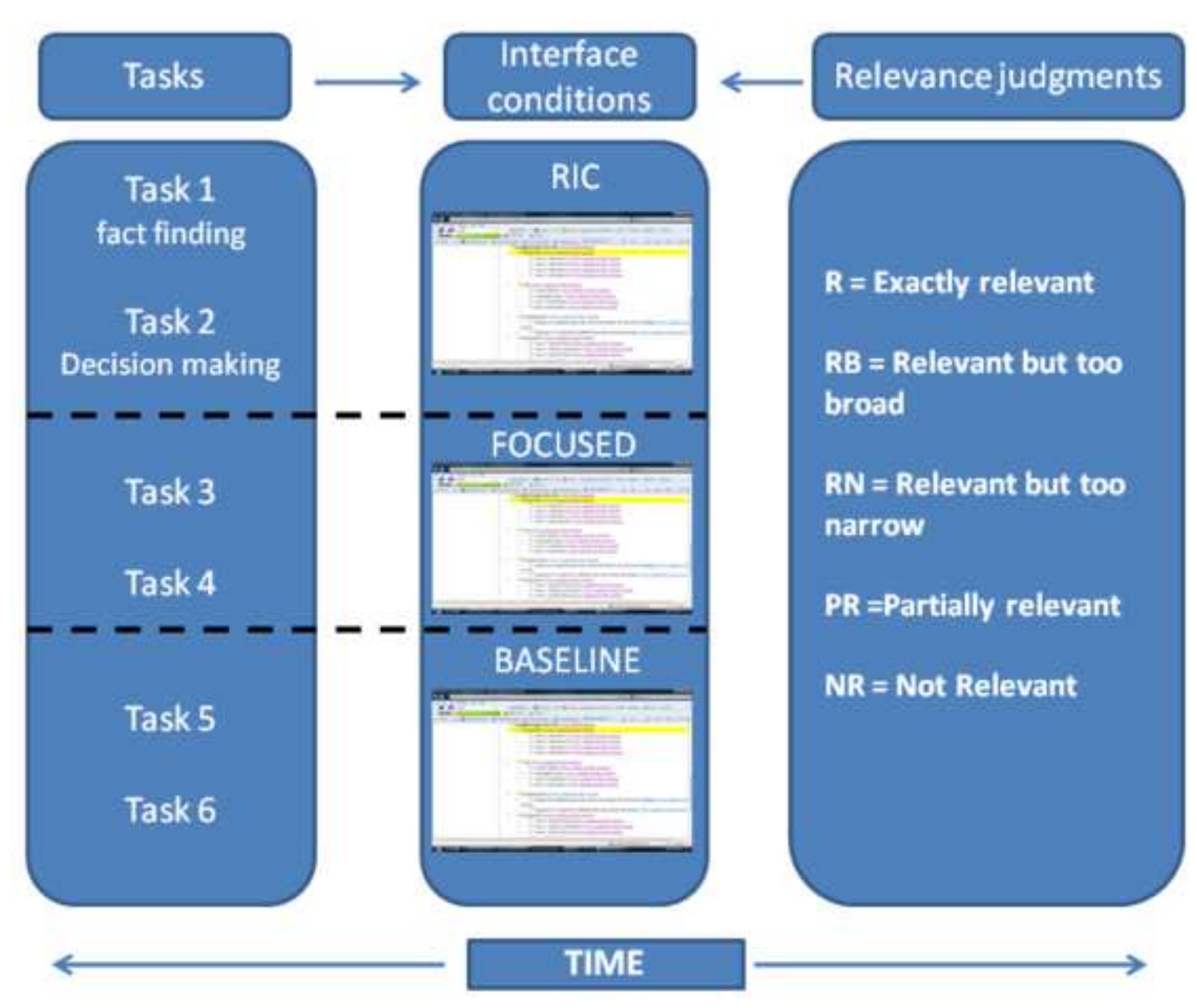




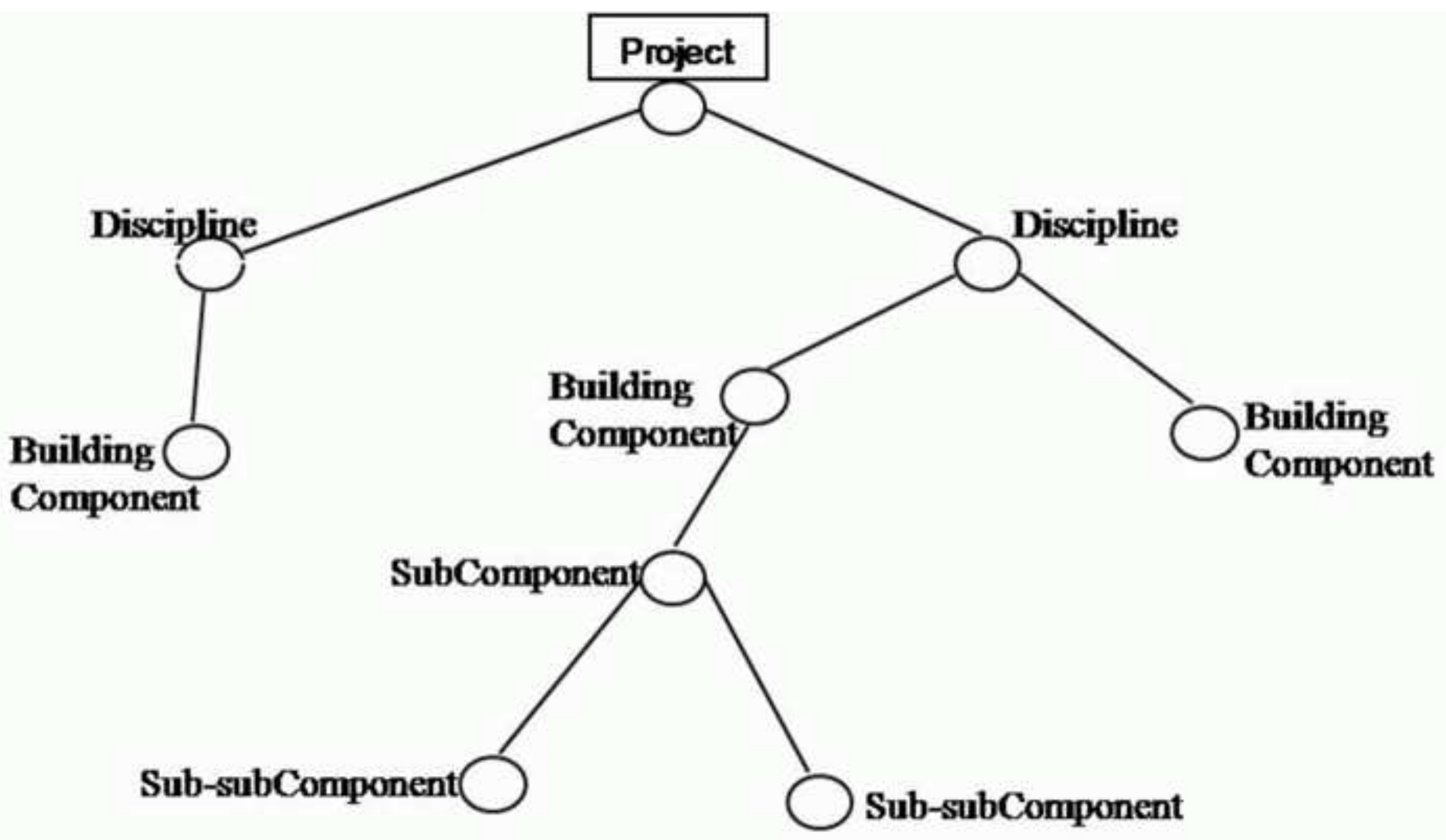




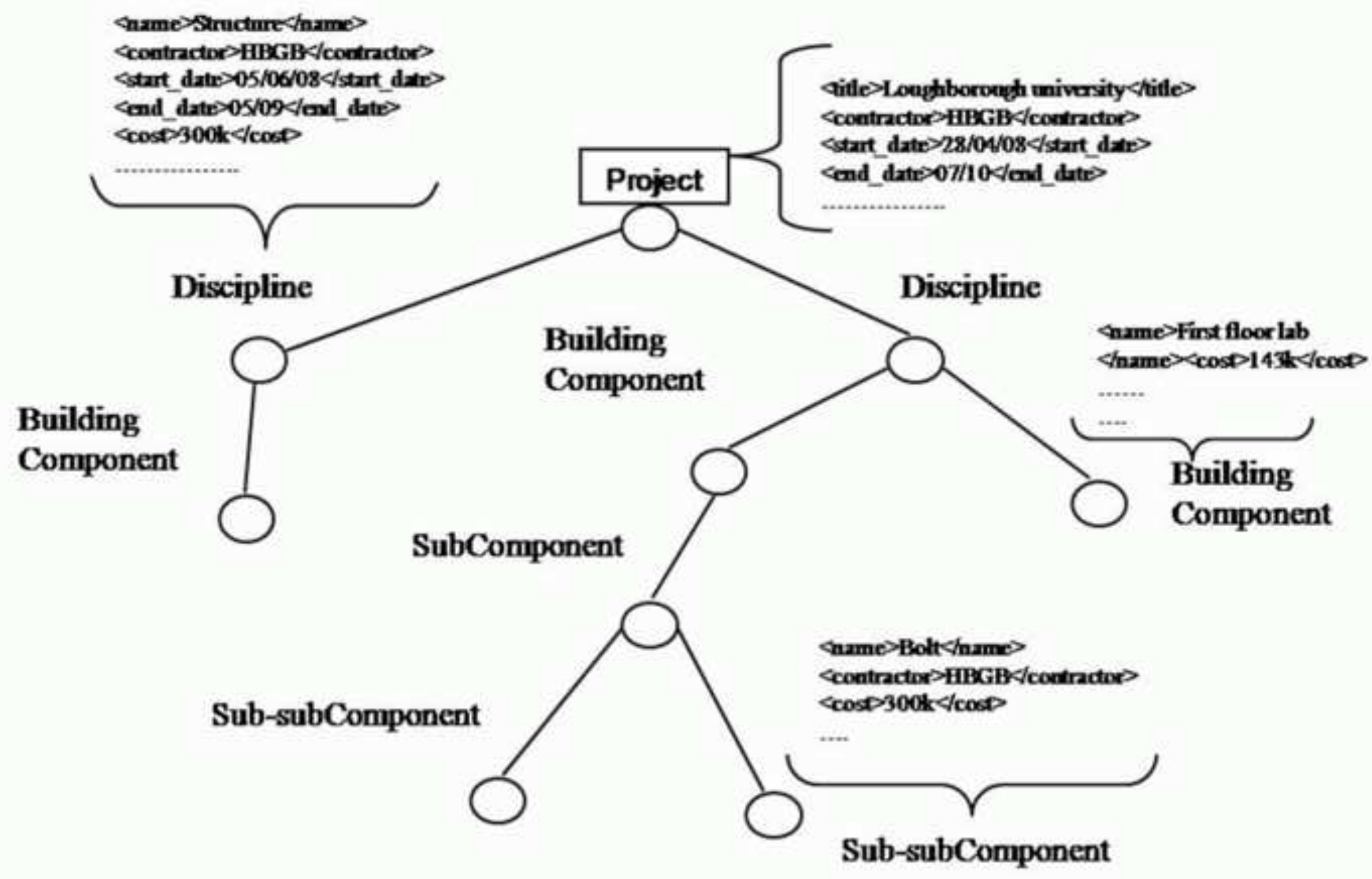




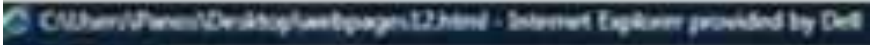

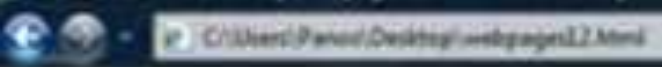

\section{Norton}

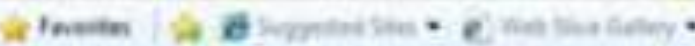

2. Ewaves

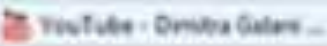

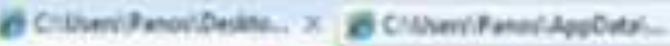

\section{CoMem-XML}

\section{Result overview: \\ Standards for steel frames in uaiversity campuses}

\section{Context}

\section{Category: Stcel fremes}

Relevant components displayed in context
Newcaslte university, Research School of Life sci Strcucture (Expand)

Roof fe (View soenponent)

First floor cabin (View component)

Q secood floor cabis (View compopent)

D First floor kitchen Diew component

Site (Fvind)

Central plant, pre finction (Central plant)

D Unassigned space (Vick composent?

D Area 1 (Laboratory) (View conwonent)

D Area 2 (Laboratory) (Virw compoerot)

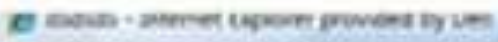

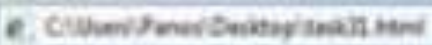

Painting the Steel Frame of a New Univenity Buildisa University of Leeds This image echoes Mare Ribosd's famous photograph of a man painting the Eiffel Tower the previous year, In the 1950 s, the University bezas at expansion progranme under the direstion of the umiver architects, Lanchester and/odge. Rather than imitate th architecture of Oxbridge had been the case with the University's earlier build Fo, the use of Portland stone a stripped down classici gave a "civic' feel to the ni: buildings. 'Lasting Mone , exhibition of the wotk of Riboud, Leeds, 1954 \& 2004. Curated by Anna Dougla Janet $\mathrm{F}$ betwec 1954

\section{New window with the actualcontent}

Is Steel frame. (Fxpard)

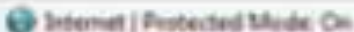
$8 \cdot 512$

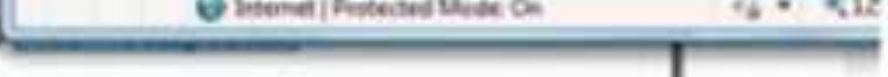




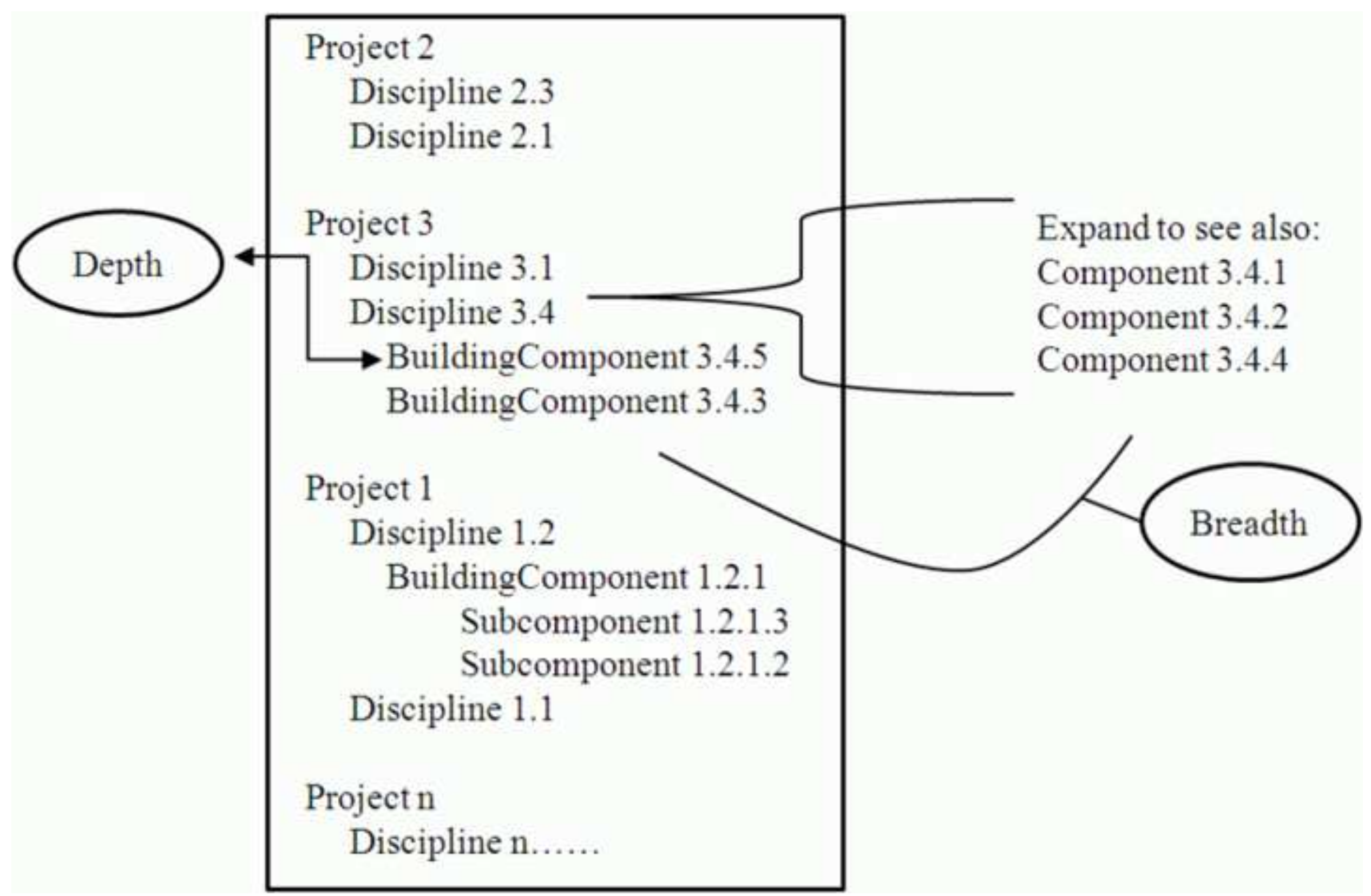


Click here to download high resolution image

BuildingComponent 2.1.1

Subcomponent 1.2

Subcomponent 5.1

Subcomponent 4.3.2

BuildingComponent 3.3.4

BuildingComponent n... 
Click here to download high resolution image

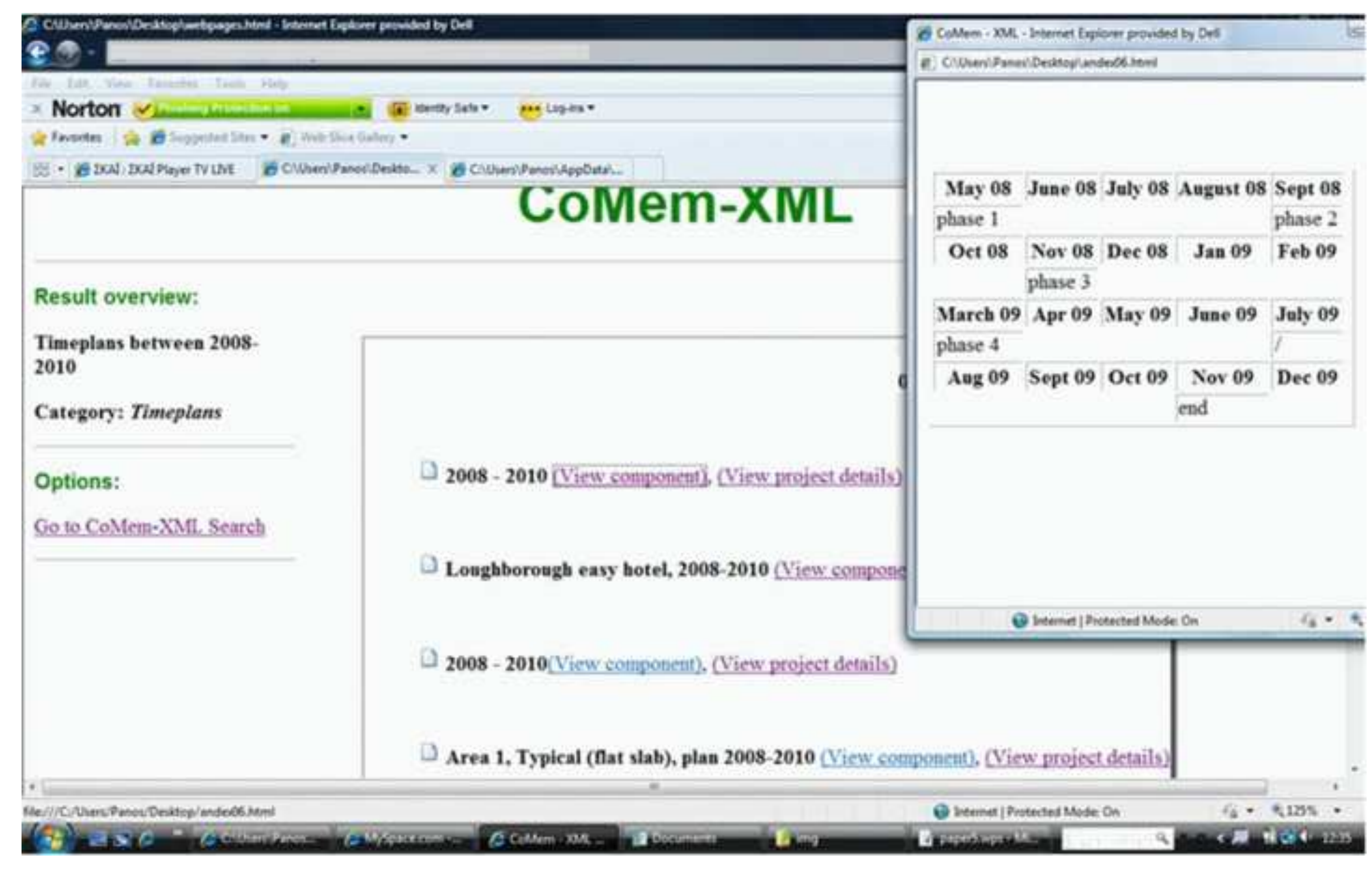

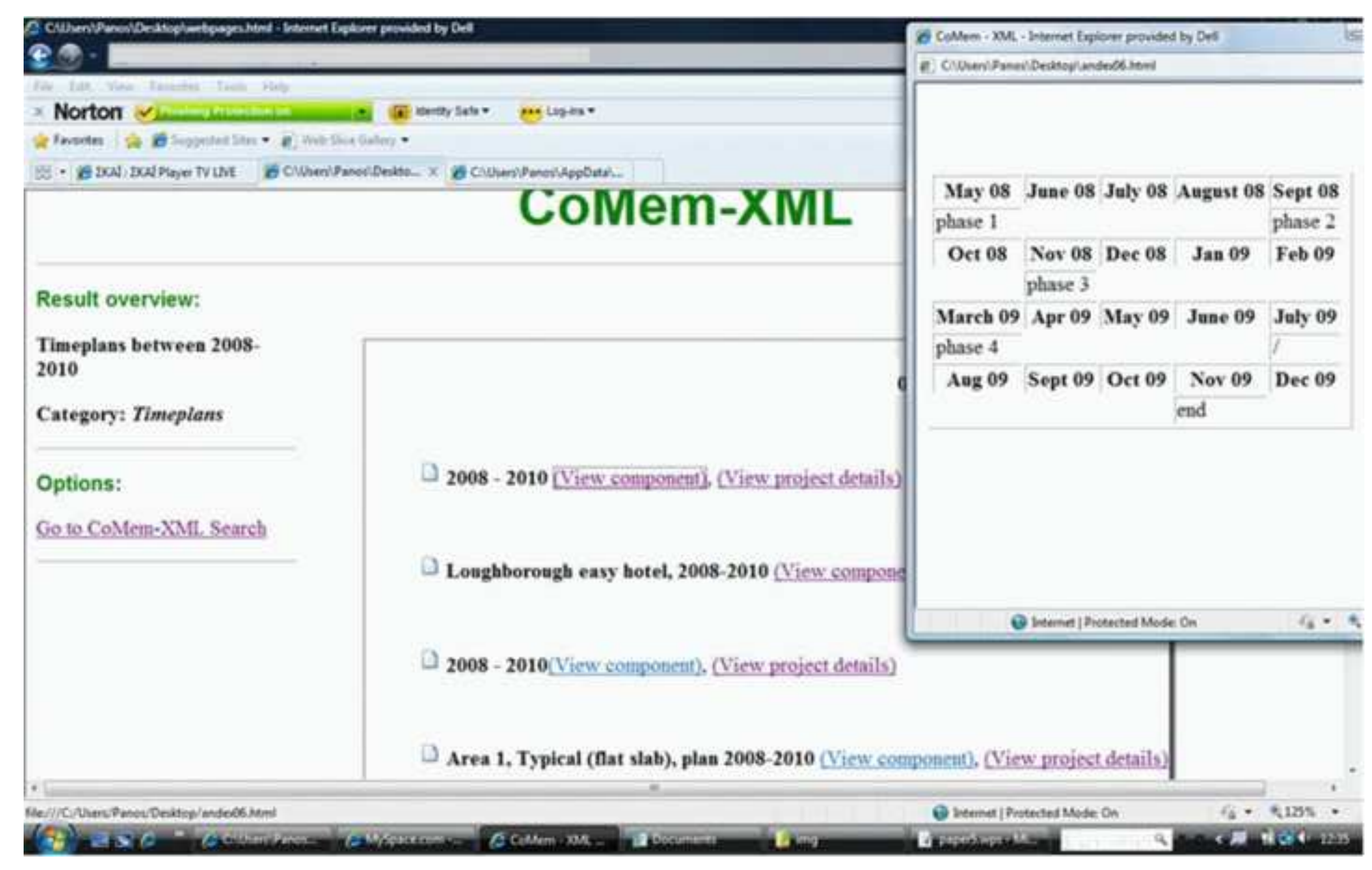

.

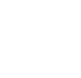

(1)
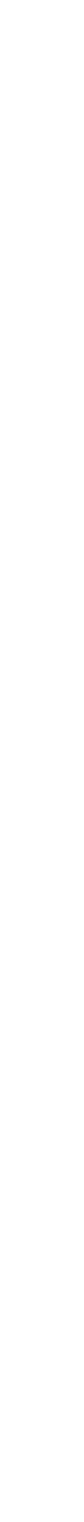

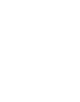




\section{Project 3}

BuildingComponent 3.1

BuildingComponent 3.2

BuildingComponent 3.3

SubComponent 3.3.1

BuildingComponent 3.4

Project 1

BuildingComponent 1.1

SubComponent 1.1.1

SubComponent 1.1.2

SubComponent 1.1.3

BuildingComponent 1.2

SubComponent 1.2.1

$\cdots$

Project 2

*.

Project 6 


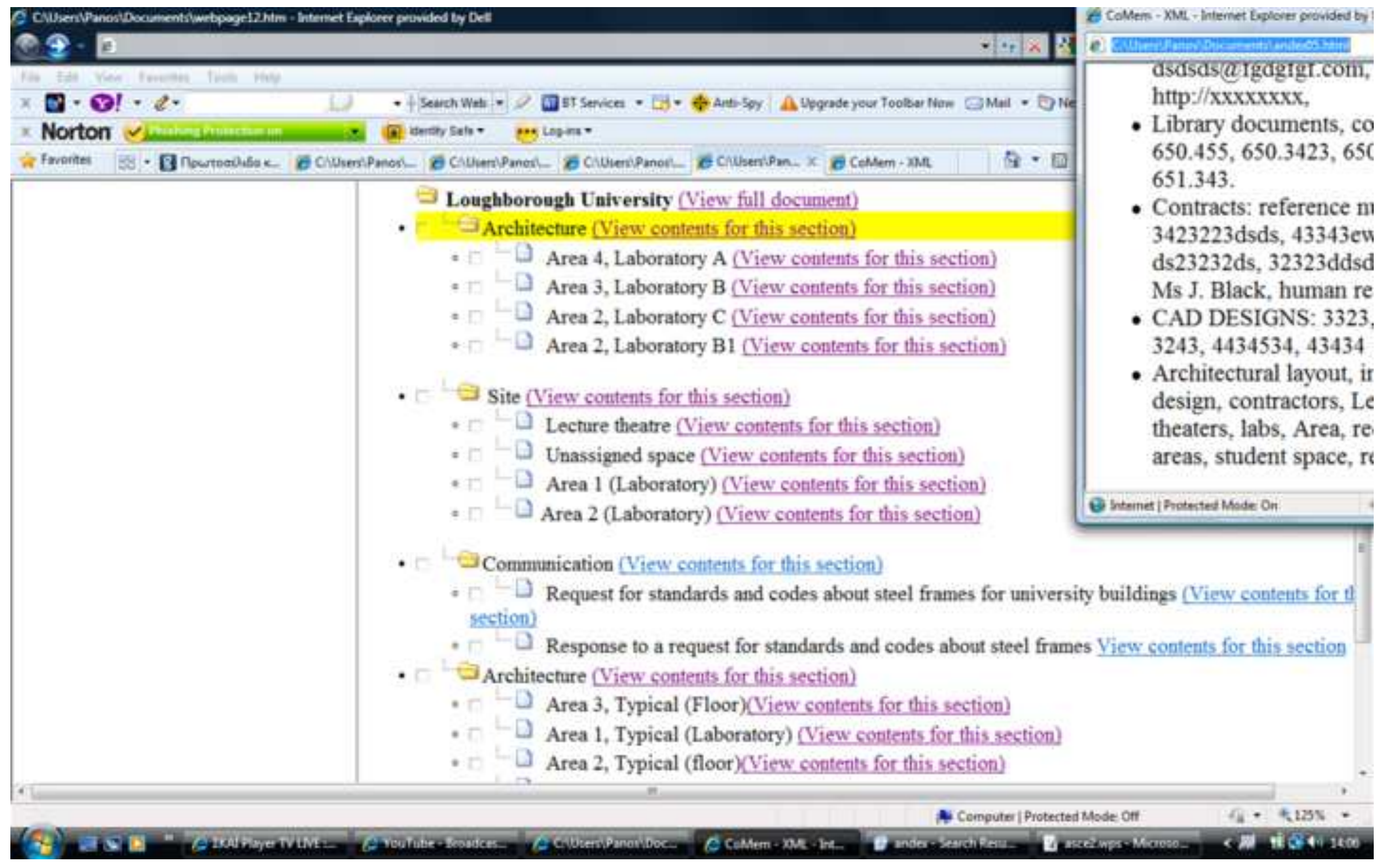


Click here to download high resolution image

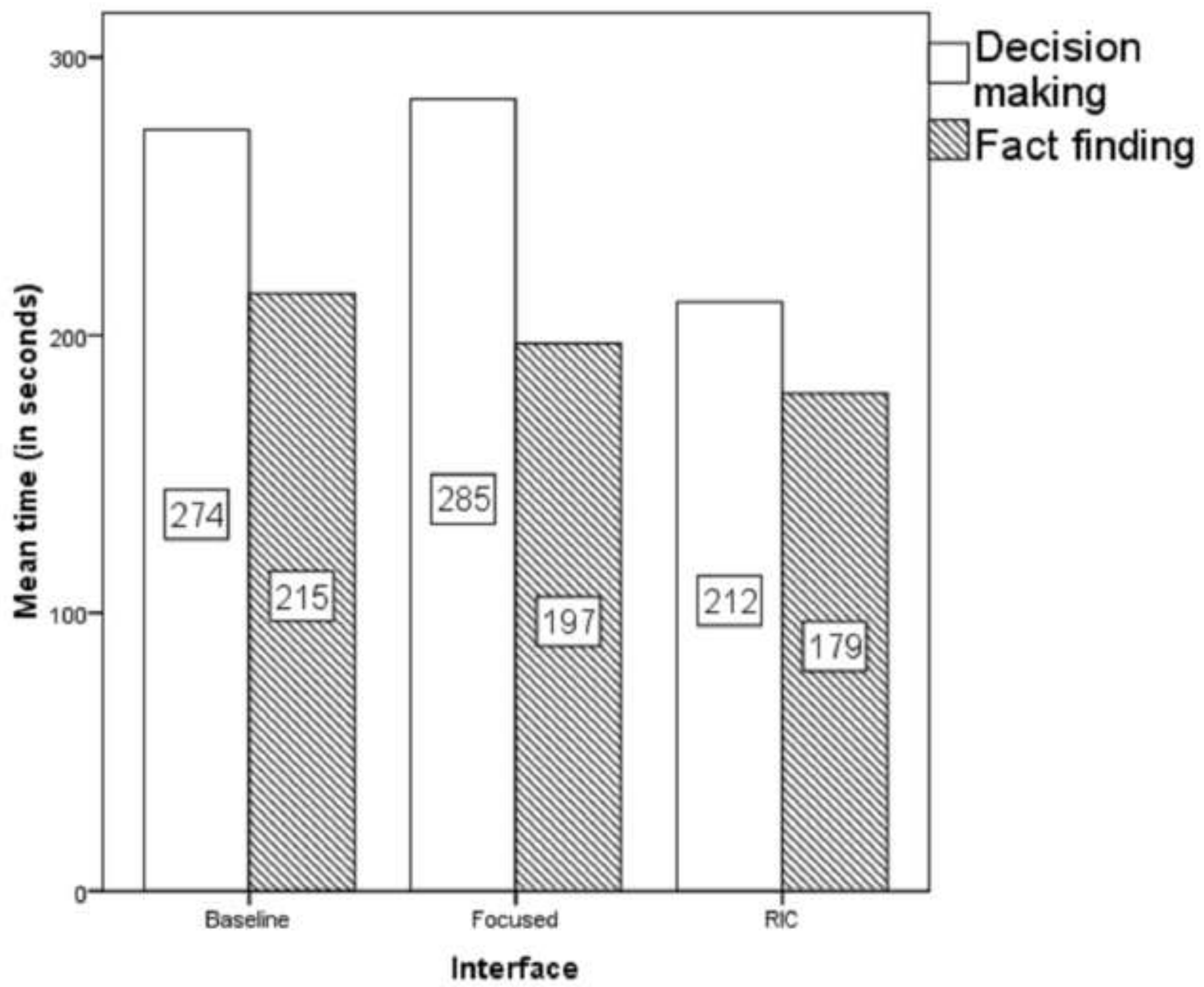




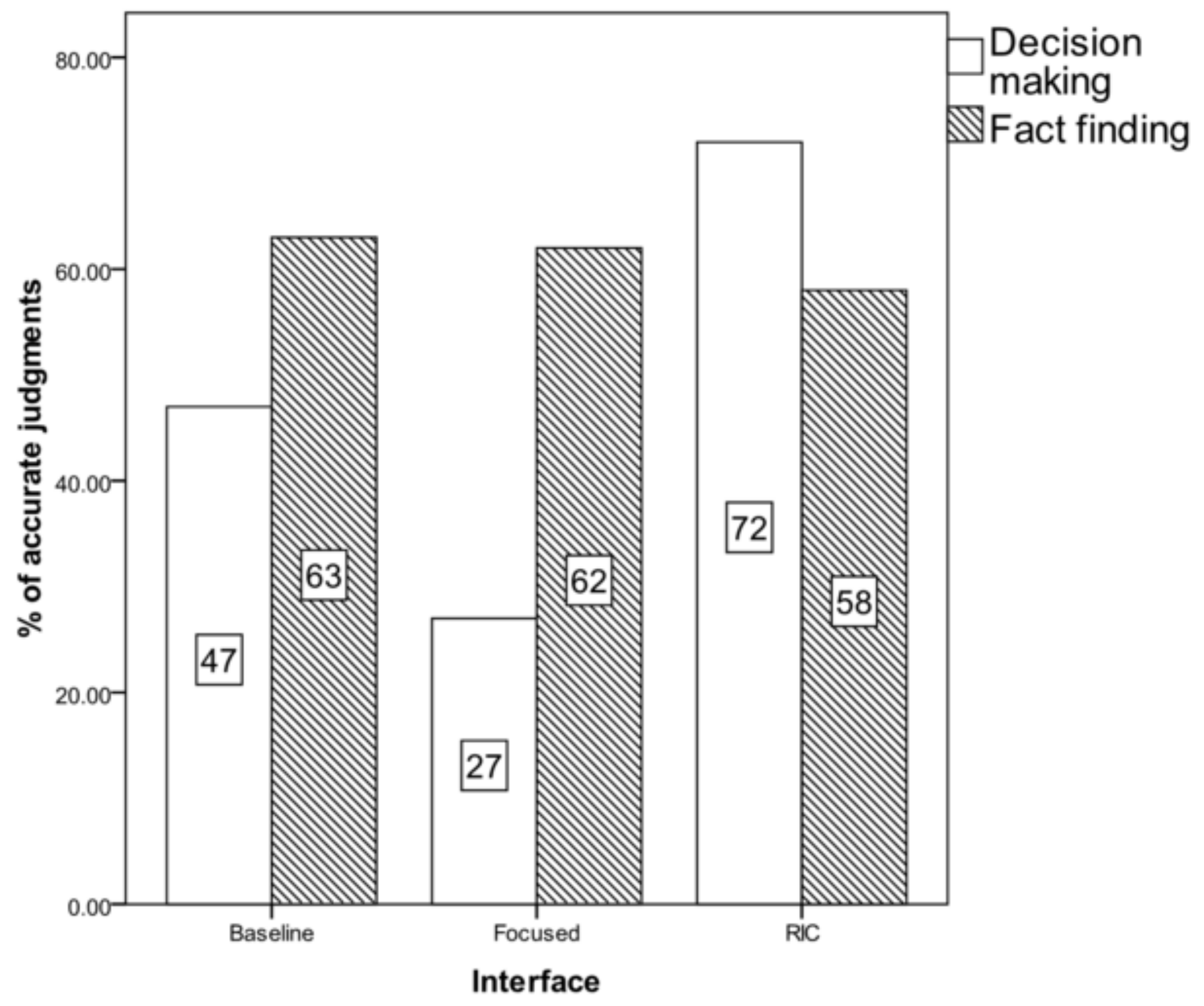

